\title{
A NOTE ON THE VIABILITY OF GASEOUS IONIZATION IN ACTIVE GALAXIES BY FAST SHOCKS
}

\author{
Ari Laor \\ Physics Department, Technion, Haifa 32000, Israel \\ laor@physics.technion.ac.il
}

\begin{abstract}
Morphological and spectroscopic evidence suggest that shocks may affect the spatial and velocity distributions of gas in the narrow line region (NLR) and extended NLR of some active galaxies. It thus seemed plausible that shocks may also energize the NLR. The observed emission line ratios strongly favor photoionization as the heating source for this gas, but it is not clear whether the ionizing radiation is generated in the NLR by "photoionizing shocks" or whether the ionizing radiation originates at the central continuum source. Here I point out that shocks are highly inefficient in producing line emission. Shocks in the NLR can convert at most $\sim 10^{-6}$ of the rest mass to ionizing radiation, compared with a maximum conversion efficiency of $\sim 10^{-1}$ for the central continuum source. The required mass flow rate through "photoionizing shocks" in the NLR is thus a few orders of magnitude higher than the mass accretion rate required to power the NLR by the central continuum source. Since gravity appears to dominate the NLR cloud dynamics, shocks must lead to an inflow, and the implied high inflow rates can be ruled out in most active galaxies.

NLR dynamics driven by a thermal wind or by some jet configurations may produce the mass flux through shocks required for photoionizing shocks to be viable, but the mass flux inward from the NLR must be kept $\sim 100-1000$ times smaller.

Photoionizing shocks are a viable mechanism in very low luminosity active galaxies if they are highly sub-Eddington $\left(\lesssim 10^{-4}\right)$ and if they convert mass to radiation with a very low efficiency $\left(\lesssim 10^{-4}\right)$.
\end{abstract}

Subject headings: galaxies: active-galaxies: Seyfert- shock waves 


\section{INTRODUCTION}

Active Galactic Nuclei (AGNs) display emission lines originating from clouds in the narrow line region (NLR) and the broad lines region (BLR; e.g. Netzer 1990). The line emission could be powered either locally through cloud-cloud collisions (Daltabuit, MacAlpine \& Cox, 1978), or by absorption of ionizing radiation from the continuum source at the center (e.g. Davidson \& Netzer 1979). The time lagged response of the broad emission lines to UV continuum variability (e.g. Peterson 1993) provides nearly conclusive evidence that the BLR clouds are powered by the central continuum source. The same relation cannot be established for the NLR since reverberation timescales are far too long. The observed line ratios in the NLR argue strongly in favor of pure photoionization (e.g. Ferland \& Osterbrock 1986), which suggests that the NLR is also powered by the central continuum source. However, some models suggest that shocks due to cloud-cloud collisions may provide significant heating for the NLR, in addition to the central continuum source (e.g. Contini \& Aldrovandi 1983, Contini 1997).

Renewed interest in shocks as a viable energy source came following the detailed calculations of Dopita \& Sutherland $(1995,1996)$ who have shown that fast shocks produce strong ionizing radiation which can be reprocessed into line emission. Thus, although the emission line spectrum from the NLR strongly suggests that clouds are powered by photoionization, rather than collisional ionization, this power could be produced by shocks in the NLR, rather than by the central continuum source. Morphological and spectroscopic evidence suggest that jet-cloud interactions may produce such shocks in the extended NLR of nearby AGNs (e.g. Cecil, Morse, \& Veilleux, 1995; Capetti, Axon, \& Macchetto 1997; Falcke, Wilson \& Simpson 1998). For a comprehensive review and a critical assessment of the available observational evidence for photoionizing shocks in AGNs see Morse, Raymond \& Wilson (1996) and Wilson (1997).

The purpose of this note is to point out that shocks are highly inefficient, compared with the central AGN, in converting mass to ionizing radiation, and can thus be ruled out as a significant source of energy for the NLR for most AGNs.

\section{QUALITATIVE ESTIMATES}

Shocks convert part of the directed kinetic energy of the colliding gas clouds to thermal energy. If all the kinetic energy of cloud-cloud collision in the NLR is converted to heat, all the heat is converted to ionizing radiation, and all the ionizing radiation is absorbed and converted to line emission, then the maximum fraction of rest mass converted to line emission would be $\epsilon_{\text {shock }}=v^{2} / 2 c^{2}$. The typical velocity of gas in the NLR is $\sim 250-500 \mathrm{~km} \mathrm{~s}^{-1}$ (see $\S 3$ ), and thus the maximum efficiency of rest mass to line emission conversion is $\epsilon_{\text {shock }} \sim 10^{-6}$.

If the gas reaches the center of the active nucleus, about $10 \%$ of its rest mass is converted to heat, which may be radiated away and converted to line emission, yielding a maximum efficiency of $\epsilon_{\mathrm{AGN}} \sim 0.1$. Thus, basic energy conservation implies that the photoionizing shocks scenario requires a mass flow through NLR shocks which is a few orders of magnitude larger than required into the central photoionizing source. A more accurate estimate of the required accretion rates in both scenarios is given below.

\section{QUANTITATIVE ESTIMATES}

The [O III $\lambda 5007$ line is typically the strongest narrow line in bright active galaxies (e.g. Ferland \& Osterbrock 1986; Veilleux \& Osterbrock 1987), and I therefore use it below as a measure of the NLR luminosity.

Dopita \& Sutherland (1995) derived the following scaling relation for the $\mathrm{H} \beta$ flux per unit area generated in the cooling gas behind a fast photoionizing shock

$$
l_{\mathrm{H} \beta}=7.44 \times 10^{-6} v_{100}^{2.41} n \quad \operatorname{ergs~} \mathrm{cm}^{-2} \mathrm{~s}^{-1},
$$

where $v=100 v_{100} \mathrm{~km} \mathrm{~s}^{-1}$ is the shock velocity and $n$ is the ambient gas density. Additional line emission is produced in the unperturbed gas in front of the shock, the "precursor H II region", which is given by

$$
l_{\mathrm{H} \beta}=9.85 \times 10^{-6} v_{100}^{2.28} n \quad \operatorname{ergs~cm}^{-2} \mathrm{~s}^{-1} .
$$

The highest [O III] $\lambda 5007 / \mathrm{H} \beta$ flux ratio found by Dopita \& Sutherland is $\sim 13$, and we therefore assume $l_{\text {[O III }]} \leq 13 l_{\mathrm{H} \beta}$. The mass flux through the shocks is

$$
\dot{M}_{\text {shock }}=A_{s} n v,
$$

where $A_{s}=L_{[\mathrm{O} \text { III }]} / l_{[\mathrm{O} \text { III }]}$ is the shock area, and $L_{[\mathrm{O} \text { III }]}$ is the intrinsic [O III $] \lambda 5007$ luminosity. Thus, 
using the shock + precursor line emission, one gets

$\dot{M}_{\text {shock }} \geq 2730 L_{42} v_{100}^{-1.41}\left(1+1.32 v_{100}^{-0.13}\right)^{-1} M_{\odot} \mathrm{yr}^{-1}$,

where $L_{[\mathrm{O} \text { III }]}=10^{42} L_{42} \operatorname{erg~s}^{-1}$.

The relation between the mass accreted by the AGN, $\dot{M}_{\mathrm{AGN}}$, and $L_{[\mathrm{O} \text { III] }}$ resulting from photoionization by the AGN is not unique. This relation depends on the accretion efficiency, the covering factor of the NLR gas, the ionization parameter and density of the NLR gas, and the presence of dust within the NLR gas. Rather than deriving a theoretical relation between $\dot{M}_{\mathrm{AGN}}$ and $L_{[\mathrm{O} \text { III] }}$, which would require knowledge of the parameters mentioned above, I use the observed $L_{[\mathrm{O} \text { III] }}$ versus $\dot{M}_{\mathrm{AGN}}$ relation. The continuum $\nu L_{\nu}$ at $5000 \AA$ is given by $L_{[\mathrm{O} \text { III }]} \times 5000 / E W_{[\mathrm{O} \mathrm{III}]}$, where $E W_{[\mathrm{O} \text { III] }}$ is the equivalent width of $[\mathrm{O}$ III] in units of $\AA$. The bolometric luminosity is $L_{\mathrm{Bol}} \sim 10 \times \nu L_{\nu}(5000 \AA)$ (e.g. fig.7 in Laor \& Draine 1993), and the accretion rate is $\dot{M}_{\mathrm{AGN}}=L_{\mathrm{Bol}} / \epsilon_{\mathrm{AGN}} c^{2}$. These relations give

$$
\dot{M}_{\mathrm{AGN}}=0.293 L_{42} E W_{30}^{-1} \epsilon_{0.1}^{-1} \quad M_{\odot} \mathrm{yr}^{-1}
$$

where $E W_{[\mathrm{O} \text { III] }}=30 E W_{30} \AA$, and $\epsilon_{\mathrm{AGN}}=0.1 \epsilon_{0.1}$.

The ratio $\dot{M}_{\text {shock }} / \dot{M}_{\mathrm{AGN}}$ is a function of $v_{100}, E W_{30}$ and $\epsilon_{0.1}$. Below we estimate typical values and dispersions for these parameters.

The $\left[\begin{array}{ll}O & I I\end{array}\right]$ FWHM data for 71 broad line active galaxies presented in Fig.6a of Whittle (1985) gives $\left\langle v_{100}\right\rangle=3.5_{-1}^{+1.4}$, where the quoted range includes $2 / 3$ of the objects (equivalent to $\pm 1 \sigma$ for a normal distribution). Data on the $E W_{[\mathrm{O} \text { III] }}$ of 36 Seyfert 1 galaxies in Osterbrock (1977) gives $\left\langle E W_{[\mathrm{O} \mathrm{III}]}\right\rangle=39 \pm 22$, and the complete sample of Boroson \& Green (1992) gives $\left\langle E W_{[\mathrm{O} \text { III }]}\right\rangle=37_{-23}^{+40}$ for 18 Seyfert 1 galaxies and $\left\langle E W_{[\mathrm{O}}\right.$ III] $\rangle=20_{-14}^{+22}$ for 69 quasars. We therefore adopt $v_{100} \sim 3.5$, and $E W_{30} \sim 1$ as typical values, which gives

$$
\frac{\dot{M}_{\text {shock }}}{\dot{M}_{\mathrm{AGN}}} \sim 750 \epsilon_{0.1},
$$

with a typical range of about a factor of two above and below.

The value of $\epsilon_{0.1}$ can be estimated by comparing the time integrated density of quasar light with the current density of massive black holes, as determined by the black hole to bulge mass correlation suggested by Magorrian et al. (1997). This comparison provides a rather strict lower limit on AGNs time average accretion efficiency of $\epsilon_{0.1} \simeq 0.09$ (see equations $1 \& 2$ in
Haehnelt, Natarajan, \& Rees 1997 with $h=0.7$ and $f_{B}=0.1$ ). Larger values for the mean observed $\epsilon_{0.1}$ are obtained if not all bulges contain massive black holes, or if some of the black holes growth occurs in an unobserved phase with $\epsilon_{0.1} \ll 1$ (see discussion in Haehnelt et al.). Typical accretion scenarios give $\epsilon_{0.1} \sim 1$. Thus, $\dot{M}_{\text {shock }}$ is at least about two, and more likely nearly three orders of magnitude larger than $\dot{M}_{\mathrm{AGN}}$.

\section{DISCUSSION}

\subsection{Gravity Powered Shocks}

If the gas velocity in the NLR is dominated by gravity, as strongly suggested by observations (Whittle 1992a, 1992b), then the loss of kinetic energy in shocks necessarily implies a loss of angular momentum, which must lead to an inflow. If there is no accumulation of mass somewhere between the central black hole and the NLR then $\dot{M}_{\text {shock }}$ eventually becomes $\dot{M}_{\mathrm{AGN}}$. Having $\dot{M}_{\text {shock }}=\dot{M}_{\mathrm{AGN}}$ requires $\epsilon_{0.1} \sim 10^{-3}$. However, the estimates of Haehnelt et al. mentioned above provide a lower limit of $\epsilon_{0.1} \simeq 0.1$ for the average efficiency. Thus, if the NLR of most AGNs is powered by shocks, then the mass flow through the NLR cannot accumulate in the central black hole. Can $\dot{M}_{\text {shock }}$ accumulate somewhere between the central black hole and the NLR?

The black hole mass estimates of Magorrian et al. and of earlier studies of the cores of normal galaxies (e.g. Kormendy \& Richstone 1995) are based on stellar velocity dispersions in the central 10-100 pc. Thus, the limits on the accretion efficiency apply on the scale of the NLR as well. Stellar velocity dispersions in a few nearby AGNs also indicate that the mass interior to the NLR is significantly smaller than expected from shock excitation (Oliva 1997). Thus, as pointed out by Oliva (1997) for the coronal line region in NGC 1068, there is no place to hide the large $\dot{M}_{\text {shock }}$ implied by the photoionizing shocks scenario for an astrophysically interesting period of time.

Cloud-cloud collisions as a source of photoionizing shocks are a viable mechanism only in low luminosity AGNs where $L / L_{E} \lesssim 10^{-4}$, such as the Galactic center (Narayan, Yi, \& Mahadevan 1995), M87 (Reynolds et al. 1996, see a photoionizing shock model in Dopita et al. 1997), and possibly LINERS (Ho, Filippenko, \& Sargent 1993), since such objects can maintain $\epsilon_{0.1} \lesssim 10^{-3}$, without accumulating excessive mass, for a non negligible fraction of their 
lifetime. The observed emission spectrum of LINERS, however, appears to argue against photoionizing shocks (Ferland \& Netzer 1983; Filippenko 1985; Ho, Filippenko, \& Sargent 1993, 1996; Maoz et al. 1995).

\subsection{Wind Powered Shocks}

Shocks in the NLR would not necessarily lead to an inflow if the gas motion is not dominated by gravity. In particular, the shocks may be produced by interaction of NLR clouds with an outflowing wind. The clouds cannot be in pure radial outflow since that would result in mass ejection at a rate $\dot{M}_{\text {shock }}$ and mass and energy conservation requires a time average $\dot{M}_{\text {shock }}<\dot{M}_{\mathrm{AGN}}$, which can be maintained only when $\epsilon_{0.1} \lesssim 10^{-3}$. One may imagine instead a "turbulent" velocity field where the required $\dot{M}_{\text {shock }} \sim 220 L_{42} M_{\odot} \mathrm{yr}^{-1}$ (for $v_{100}=3.5$ ) flows in and out within the NLR, with only $0.1-1 \%$ of this accretion rate being able to flow further inward to provide $\dot{M}_{\mathrm{AGN}}$.

What can power such a wind? One possibility may be a radiation-pressure driven wind (e.g. on dust grains). The maximum momentum flux available in this case is $\dot{P}_{\mathrm{rad}}=L_{\mathrm{Bol}} / c$, or $\dot{P}_{\mathrm{rad}}=\dot{M}_{\mathrm{AGN}} \epsilon_{\mathrm{AGN}} c$. The momentum flux in shocked gas is $\dot{P}_{\text {shock }}=$ $\dot{M}_{\text {shock }} v$, and the maximum ratio between available and required momentum fluxes,

$$
\dot{P}_{\text {rad }} / \dot{P}_{\text {shock }}=0.1 \epsilon_{0.1} c / 750 \epsilon_{0.1} v
$$

is about an order of magnitude too small.

Another possibility is a thermal-pressure driven wind, in which case momentun is not conserved locally, but only globaly. What can heat this wind? The wind velocity should be of the order of $250-500 \mathrm{~km} \mathrm{~s}^{-1}$ which corresponds to $T \sim 1-3 \times 10^{7} \mathrm{~K}$. This temperature range is obtained naturally for Compton-heated winds in AGNs (Begelman, McKee, \& Shields 1983; Mathews \& Ferland 1987). The photoionizing energy flux in the NLR is $E_{\mathrm{ph}} n_{\mathrm{ph}} c$, where $E_{\mathrm{ph}} \sim 30 \mathrm{eV}$ is the mean photon energy, and $n_{\mathrm{ph}}$ is the photon density. The mechanical energy flux provided by the wind is $E_{\mathrm{p}} 2 n c$, where $E_{\mathrm{p}} \sim 1 \mathrm{keV}$ is the mean particle energy. The photoionizing/mechanical flux ratio is thus $13 U$, where $U \equiv n_{\mathrm{ph}} / n$ is the wind ionization parameter. The Compton temperature is obtained for $U>100$, and thus mechanical (i.e. shock) heating would be a negligible effect compared with direct photoionization heating.

Alternatively, a thermal wind may be mechanically heated by the dissipation of a jet kinetic en- ergy, as seen for example in jet cocoons. The rate of energy deposition by the wind into the shocks is roughly $\frac{3}{16} \dot{M}_{\text {shock }} v^{2}=3.2 \times 10^{42} L_{42}$ erg $\mathrm{s}^{-1}$ (for $v_{100}=3.5$ ), which should be provided by the jet. Since $L_{\mathrm{Bol}}=1.7 \times 10^{45} L_{42} E W_{30}^{-1}$, the jet requires only $\sim 0.2 \%$ of $L_{\mathrm{Bol}}$. The energy deposited in the shocked gas should alos be observable as an extended non-variable source of continuum emission in the optical to soft X-ray range. Additional spatially extended free-free emission at $T \sim 1-3 \times 10^{7} \mathrm{~K}$ should come from the thermal wind, but the luminosity of this component could be well below $10^{42} \mathrm{erg} \mathrm{s}^{-1}$.

\subsection{Jet Powered Shocks}

Although gravity appears to play the key role in the NLR, Whittle (1992a, 1992b) finds evidence for excess velocity dispersions in a small fraction of AGNs (those with a high radio luminosity and linear radio morphology), which can be attributed to a jet-cloud interaction (see also Bicknell et al. 1997). Thus one can imagine a picture where clouds are swept up by the jet, and later fall back in, with nearly zero net accretion rate through the NLR, just as in the wind powered shocks picture.

The jet starts at the center with velocity $v_{\text {jet }}$ and mass flux $\dot{M}_{\text {jet }}$, gradually entraining local gas until its mass flux increases to $\dot{M}_{\text {shock }}$ and its velocity drops to $v$. Mass, momentum, and energy conservation are used below to constrain the properties of such a jet. The jet motion is assumed to be balistic and any possible interaction with a confining medium is neglected.

Momentum flux conservation gives

$$
\dot{M}_{\text {jet }}=v \dot{M}_{\text {shock }} / \gamma_{\text {jet }} \beta_{\text {jet }} c \text {, }
$$

where $\beta_{\text {jet }}=v_{\text {jet }} / c$ and $\gamma_{\text {jet }}=\left(1-\beta_{\text {jet }}^{2}\right)^{-1 / 2}$ (note that $\left.\beta_{\text {shock }} \lesssim 0.0017, \gamma_{\text {shock }} \sim 1\right)$. Mass flux conservation requires $\dot{M}_{\text {jet }}<\dot{M}_{\mathrm{AGN}}$ which together with the above expression gives

$$
\gamma_{\text {jet }} \beta_{\text {jet }} c / v>\dot{M}_{\text {shock }} / \dot{M}_{\mathrm{AGN}} .
$$

Using the $\dot{M}_{\text {shock }} / \dot{M}_{\text {AGN }}$ relation obtained in $\S 3$ (note that this relation is independent of the presence of a jet), and $v=350 \mathrm{~km} \mathrm{~s}^{-1}$ gives $\gamma_{\text {jet }} \beta_{\text {jet }}>0.875 \epsilon_{0.1}$ or

$$
\beta_{\text {jet }}>0.875 \epsilon_{0.1} / \sqrt{1+0.77 \epsilon_{0.1}^{2}} .
$$

This is the minimum jet velocity which can provide the required momentum flux without exceeding the mass flux available through accretion. 
The flux of kinetic energy in the jet must be smaller than the energy flux generated by the accretion, i.e.

$$
\left(\gamma_{\mathrm{jet}}-1\right) \dot{M}_{\mathrm{jet}} c^{2}<0.1 \epsilon_{0.1} \dot{M}_{\mathrm{AGN}} c^{2} .
$$

Substituting for $\dot{M}_{\text {jet }}$ from the momentum flux conservation, and using the $\dot{M}_{\text {shock }} / \dot{M}_{\mathrm{AGN}}$ relation obtained in $\S 3$ gives

$$
0.1 \epsilon_{0.1} \gamma_{\text {jet }} \beta_{\text {jet }} c /\left(\gamma_{\text {jet }}-1\right) v>750 \epsilon_{0.1}
$$

which for $v=350 \mathrm{~km} \mathrm{~s}^{-1}$ gives

$$
\beta_{\text {jet }}<0.26
$$

This is the maximum jet velocity which can provide the required momentum flux without exceeding the energy flux available through accretion. The upper and lower limits on $\beta_{\text {jet }}$ obtained above allow a solution for $\beta_{\text {jet }}$ only if $\epsilon_{0.1}<0.26$. Thus, for jet entrainment to be viable the jet must be subrelativistic, and the accretion efficiency must be fairly low.

As the jet entrains ambient gas it slows down, while conserving momentum and losing kinetic energy. The lost kinetic energy is converted to internal energy of the entrained gas and some of it will be radiated away. For example, in a jet with a solid angle of $\Omega / 4 \pi=0.01$, reaching a final $v_{100}=3.5$, i.e. $\dot{M}_{\text {shock }}=220 L_{42} M_{\odot} \mathrm{yr}^{-1}$, at $r=10 \mathrm{pc}$, and $T=10^{7} \mathrm{~K}$, the free-free cooling time is $\sim 6 \mathrm{yr}$, which is well below the dynamical time scale, $r / v \sim 3000 \mathrm{yr}$. The jet cooling will produce an extended continuum source, in addition to that within the shocked gas itself ( $\$ 4.2)$. This continuum can be quite intense. The initial jet kinetic energy is $L_{\text {jet }}^{\text {in }} \simeq \frac{\beta_{\text {jet }}}{0.26} L_{\mathrm{Bol}}$, the accretion efficiency is $\epsilon_{0.1}>0.1$ ( $(4.1)$, which gives a minimum jet velocity of $\beta_{\text {jet }} \simeq 0.087$ (from momentum conservation). Since the final jet kinetic energy is only $L_{\text {jet }}^{\mathrm{f}} \simeq 0.005 L_{\mathrm{Bol}}$, the dissipated kinetic energy in the entrained gas is large $\sim 0.3 L_{\mathrm{Bol}}$. A significant fraction of this internal energy is likely to be radiated through thermal and nonthermal continuum emission somewhere in the X-ray to radio bands. Thus, observational constraints on the amount of spatially extended continuum emission in nearby AGNs can be used to constrain the feasibility of the jet-cloud interaction scenario. Wilson, Ward \& Haniff (1987) find that the extended radio power in nearby AGNs is $\leq 10^{-2} L_{\mathrm{NLR}}$. Comparable constraints in other bands would rule out jet entrained gas as the source of power for the NLR in most AGNs.
If the jet is still confined as it entrains ambient gas, if cooling is negligible, and if the jet cross section increases outward, then the random particle motion can be converted back to directed motion. The jet basically converts kinetic energy to internal energy and back to kinetic energy, conserving kinetic energy and not conserving momentum (due to the confining medium). The limits obtained above will not be valid (see Phinney 1983 for a comprehensive discussion), and the jet just needs to provide the kinetic energy deposited in the shocks $\left(0.002 L_{\mathrm{Bol}}\right)$, as in the case of the thermal pressure driven wind discussed above.

A jet may also power the NLR by directly dumping its kinetic energy into gas clouds in the NLR, rather than by inducing gas motion which results in photoionizing shocks, as assumed above. However, in this case the line emission would result from thermal excitation, rather than photoionization, which is not consistent with the observed spectrum from the NLR.

\section{CONCLUSIONS}

Photoionizing shocks due to cloud-cloud collisions in a gravity dominated NLR cannot provide the source of energy for narrow line emission in most AGNs.

NLR dynamics dominated by forces other than gravity may allow the high mass flux through shocks required for photoionizing shocks to be viable. The clouds velocities can be produced by a wind-cloud interaction, which requires a mechanically heated thermal wind. Alternatively, the clouds velocities may be dominated by a jet-cloud interaction. This either implies a strong extended continuum source, which is not yet observed, or otherwise specific jet configurations. In any non-gravitational cloud dynamics picture the mass flux inward of the NLR must be kept $\sim 100-1000$ times smaller than through the NLR shocks.

Photoionizing shocks could be relevant in very low luminosity AGNs, such as LINERs, if these objects are both highly sub Eddington $\left(L / L_{E} \lesssim 10^{-4}\right)$ and if they convert mass to radiation with a very low efficiency $\left(\epsilon_{\mathrm{AGN}} \lesssim 10^{-4}\right)$.

Thoughtful and constructive comments by Sterl Phinney, Dani Maoz, and the referee are greatly appreciated. This research was supported in part by a grant from the Israel Science Foundation, by the E. and J. Bishop research fund, and by the Milton and 
Lillian Edwards academic lectureship fund.

\section{REFERENCES}

Begelman, M. C., McKee, C. F., \& Shields, G. A. 1983, ApJ, 271, 70

Bicknell, G. V., Dopita, M. A., Tsvetanov, Z. I., \& Sutherland, R. S. 1997, ApJ, submitted astroh/9709037)

Boroson, T. A., \& Green, R. F., 1992, ApJS, 80, 109

Capetti A., Axon D. J., \& Macchetto, F. D. 1997, ApJ, 487, 560

Cecil, G., Morse, J. A., \& Veilleux, S. 1995, ApJ, 452, 613

Contini, M. 1997, A\&A, 323, 71

Contini, M., \& Aldrovandi, S. M. V. 1983, A\&A, 127, 15

Daltabuit, E., MacAlpine, G. M., \& Cox, D. P. 1978 ApJ, 219, 372

Davidson, K., \& Netzer, H. 1979, Rev. Mod. Phys., 51,715

Dopita, M. A., \& Sutherland, R. S. 1995, ApJ, 455, 468

Dopita, M. A., \& Sutherland, R. S. 1996, ApJS, 102, 161

Dopita, M. A. et al. 1997, ApJ, 490, 202

Falcke, H., Wilson, A. S., \& Simpson, C., 1998, ApJ, in press

Ferland, G. J., \& Netzer, H. 1983, ApJ, 264, 105

Ferland, G. J., \& Osterbrock, D. E. 1986, ApJ, 300, 658

Filippenko, A. V., 1985, ApJ, 289, 475

Haehnelt, M. G., Natarajan, P., \& Rees, M. J. 1998, MNRAS, in press astro-ph/9712259

Ho, L. C., Filippenko, A. V., \& Sargent, W. L. W. 1993, ApJ, 417, 63

— 1996, ApJ, 462, 183

Kormendy, J., \& Richstone, D. 1995, ARA\&A, 33, 581

Laor, A., \& Draine, B. T. 1993, ApJ, 402, 441

Magorrian, J. et al. 1998, AJ, in press astrohh/9708072)

Maoz, D. et al., 1995, ApJ, 440, 91
Mathews, W. G., \& Ferland, G. J. 1987, ApJ, 323, 456

Morse, J. A., Raymond, J. C., \& Wilson, A. S. 1996, PASP, 108, 426

Narayan, R., Yi, I., \& Mahadevan, R. 1995, NATURE, 374, 623

Netzer, H. 1990, in Active Galactic Nuclei, ed. T.J.L. Courvoisier and M. Mayor (Springer: Berlin)

Oliva, E. 1997, in IAU Colloq. 159, Emission Lines in Active Galaxies: New Methods and Techniques, Eds. B. M. Peterson, F.-Z. Cheng, and A. S. Wilson (ASP, SF), 288.

Osterbrock, D. E. 1977, ApJ, 215, 733

Peterson, B. M, 1993, PASP, 105, 247

Phinney, E. S. 1983, Ph.D. thesis, University of Cambridge

Reynolds, C. S., Di Matteo, T., Fabian, A. C., Hwang, U., \& Canizares, C. R. 1996, MNRAS, 283, L111

Veilleux, S., \& Osterbrock, D. E. 1987, ApJS, 63, 295

Whittle, M. 1985, MNRAS, 213, 1

Whittle, M. 1992a, ApJ, 387, 109

Whittle, M. 1992b, ApJ, 387, 121

Wilson, A. S. 1997, in IAU Colloq. 159, Emission Lines in Active Galaxies: New Methods and Techniques, Eds. B. M. Peterson, F.-Z. Cheng, and A. S. Wilson (ASP, SF), 264.

Wilson, A. S., Ward, M. J., \& Haniff, C. A. 1988, ApJ, 334, 121

This 2-column preprint was prepared with the AAS LATEX macros $\mathrm{v} 4.0$. 